\title{
Schutz durch Mund-Nasen Schutz - Meinungen, Fort- und Weiterbildung, Wissenschaft
}

Ein Beitrag aus dem Heft 3 des Jahrgangs 2020 der Krankenhaushygiene up2date [1] kursiert derzeit ganz untypisch für unsere Zeitschrift in „Laienkreisen“ und erreicht ungewohnte Clickzahlen im Internet. Ein Grund zum Jubeln? Leider nein: Der Diskussionsbeitrag wird als „Beweis“ gegen die Maskenpflicht hergenommen und politisch instrumentalisiert. Ist hier etwas falsch gelaufen oder ist dieser Beitrag sozusagen unschuldig „unter die Räuber gefallen“?

Aus heutiger Sicht würden wir sagen: Der Beitrag hätte klar als Diskussions- und Meinungsbeitrag und nicht als CME-Artikel erscheinen sollen und wäre besser von einem entsprechenden Editorial oder einer Zweitmeinung begleitet worden - allerdings war zum Zeitpunkt des Entstehens des Beitrages die politische Dimension der „Maskenfrage“ noch nicht absehbar.

Grundsätzlich stehen jeder Autor und jede Autorin für den Inhalt ihres veröffentlichten Artikels persönlich ein und die Artikel spiegeln nicht die Sicht oder gar die Meinung des Herausgeberteams wider. Das führt uns zu der Frage: Ist Meinung in der Fort- und Weiterbildung und in der Wissenschaft überhaupt gefragt? Wir meinen, ja! Als Herausgeber wollen wir auch in Zukunft meinungsstarke Beiträge: Sie fördern den erforderlichen Prozess der kontroversen Auseinandersetzung mit wichtigen Themen, Theorien und Thesen in der Wissenschaft ebenso wie in der Fortund Weiterbildung. Dieser notwendige Diskurs muss in einem vernünftigen Rahmen, z. B. in Zeitschriften wie dieser, stattfinden und darf nicht für politische Zwecke missbraucht werden, auch wenn eine provokante Überschrift auf den ersten Blick fälschlicherweise dazu einlädt. Woran wir arbeiten müssen: Es muss jederzeit klar sein, was Wissenschaft ist, über die man mit definierten und geeigneten Mitteln streiten muss, was gesichertes Wissen in der Fort- und Weiterbildung ist, über das man nicht mehr allzu viel streiten sollte, und was Meinung ist, über die man auf sachlicher Ebene und in begrenztem Rahmen streiten kann. Ein weiterer Meinungsbeitrag liegt mittlerweile mit dem Leserbrief in Heft 4 des Jahrgangs 2020 [2] vor.

Die Herausgeber

\section{Literatur}

[1] Kappstein I. Mund-Nasen-Schutz in der Öffentlichkeit: Keine Hinweise für eine Wirksamkeit. Krankenhaushygiene up2date 2020; 15: 279-295

[2] Leiß O. Leserbrief zum Beitrag: Kappstein I. Mund-Nasen-Schutz in der Öffentlichkeit: Keine Hinweise für eine Wirksamkeit. Krankenhaushygiene up2date 2020; 15: 327 - 332 